\title{
No observed association for mitochondrial SNPs with preterm delivery and related outcomes
}

\author{
Brandon W. Alleman' ${ }^{1}$, Solveig Myking ${ }^{2}$, Kelli K. Ryckman' ${ }^{1}$ Ronny Myhre'2, Eleanor Feingold ${ }^{3}$, Bjarke Feenstra4, \\ Frank Geller ${ }^{4}$, Heather A. Boyd ${ }^{4}$, John R. Shaffer ${ }^{3}$, Qi Zhang ${ }^{5}$, Ferdouse Begum 6 , David Crosslin ${ }^{5}$, Kim Doheny $^{7}$, \\ Elizabeth Pugh7 ${ }^{7}$, Aase Serine Devold Pay², Ingrid H.G. Østensen², Nils-Halvdan Morken², Per Magnus², \\ Mary L. Marazita ${ }^{3}$, Bo Jacobsson ${ }^{2}$, Mads Melbye ${ }^{4}$ and Jeffrey C. Murray ${ }^{1}$; for the Gene, Environment Association Studies \\ (GENEVA) Consortium and Norwegian Mother and Child Cohort Study (MoBA) Genome-Wide Association Study Group
}

BACKGROUND: Preterm delivery (PTD) is the leading cause of neonatal morbidity and mortality. Epidemiologic studies indicate recurrence of PTD is maternally inherited, creating a strong possibility that mitochondrial variants contribute to its etiology. This study examines the association between mitochondrial genotypes and PTD and related outcomes.

METHODS: This study combined, through meta-analysis, two case-control, genome-wide association studies: one from the Danish National Birth Cohort Study and one from the Norwegian Mother and Child Cohort Study (MoBa) conducted by the Norwegian Institute of Public Health. The outcomes of PTD $(\leq 36 w k)$, very PTD $(\leq 32 w k)$, and preterm prelabor rupture of membranes (PPROM) were examined. A total of 135 individual single-nucleotide polymorphism (SNP) associations were tested using the combined genome from mothers and neonates (case vs. control) in each population and then pooled via meta-analysis.

RESULTS: After meta-analysis, there were four SNPs for the outcome of PTD below $P \leq 0.10$ and two below $P \leq 0.05$. For the additional outcomes of very PTD and PPROM, there were three and four SNPS, respectively, below $P \leq 0.10$.

CONCLUSION: Given the number of tests, no single SNP reached study-wide significance $(P=0.0006)$. Our study does not support the hypothesis that mitochondrial genetics contributes to the maternal transmission of PTD and related outcomes.

P reterm delivery (PTD; gestational age (GA) at delivery $<37 \mathrm{wk}$ ) and its effects are the leading causes of neonatal morbidity and mortality, responsible for $\sim 35 \%$ of infant deaths in the United States (1) and $28 \%$ of the 4 million neonatal deaths worldwide (2). Neonates who survive are at increased risk for blindness, deafness, cerebral palsy, and cognitive delay $(3,4)$. These disorders have onset in the neonatal period, and thus cause more disability-adjusted life years than adult-onset diseases. Of concern is that rates of PTD in the United States have been rising, peaking in 2006 at $12.8 \%$ overall $(11.1 \%$ in singleton births) with only slight declines since (5).

Contributing factors to PTD are many and varied. Environmental factors including smoking (6) and other substance abuse, low socioeconomic status (7), maternal stress (8), extremes in maternal age $(9,10)$, and previous adverse pregnancy outcomes (11) contribute to PTD. Moreover, physiological risk factors such as uterine anomalies (12), abnormal cholesterol (13), low vitamin D (14,15), maternal infection (16), and elevated inflammatory states (17) put delivering women at risk. The multifactorial nature of this disease makes it difficult to establish broadly applicable principles about its etiology.

Underlying the established risk factors is the role genetic predisposition plays in PTD (18). A familial component has been established with genetic studies showing that the heritability of PTD is up to $40 \%$ (19). In addition, associations of PTD and single-nucleotide polymorphisms (SNPs) in the nuclear genome have been reported (20-22). An underexplored, yet highly plausible, cause of the matrilineal risk for PTD is the role of the mitochondrial genome. Epidemiologic studies in two European populations indicate that recurrence of PTD is maternally associated, suggesting that mitochondrial variants may contribute to risk (23-25). Specific case-control studies have pointed to association within the mitochondrial genome as well (26). These indications of a mitochondrial component to PTD call for a comprehensive, genome-wide approach to identifying associated mitochondrial SNPs implicated in PTD (27).

This study examines the link between mitochondrial genome variation, which is maternally inherited, and PTD by examining SNPs from across the mitochondrial genome. This study combines results from two mitochondria-wide scans: one from a Danish population and one from a Norwegian population.

The first three and last four authors contributed equally to this work.

'Department of Pediatrics, University of lowa, lowa City, lowa; ${ }^{2}$ Division of Epidemiology, Department of Genes and Environment, Norwegian Institute of Public Health, Oslo, Norway; ${ }^{3}$ Department of Human Genetics, University of Pittsburgh, Pittsburgh, Pennsylvania; ${ }^{4}$ Department of Epidemiology, Statens Serum Institut, Copenhagen, Denmark; ${ }^{5}$ Department of Biostatistics, University of Washington, Seattle, Washington; ${ }^{6}$ Department of Biostatistics, University of Pittsburgh, Pittsburgh, Pennsylvania; ${ }^{7}$ Institute of Genetic Medicine, Johns Hopkins University School of Medicine, Baltimore, Maryland. Correspondence: Jeffrey C. Murray (jeff-murray@uiowa.edu) 
Table 1. Demographic data

\begin{tabular}{lcccccc}
\hline & \multicolumn{2}{c}{ Denmark } & & \multicolumn{2}{c}{ Norway } \\
\cline { 2 - 3 } \cline { 5 - 6 } Outcome & Cases & Controls & & Cases & Controls \\
\hline PTD & 1,307 & 988 & & 981 & 983 \\
Very PTD & 252 & 988 & & 95 & 983 \\
PPROM & 379 & 988 & & 287 & 983 \\
\hline
\end{tabular}

Cases and controls for both the Danish and Norwegian populations.

PPROM, preterm prelabor rupture of membranes; PTD, preterm delivery.

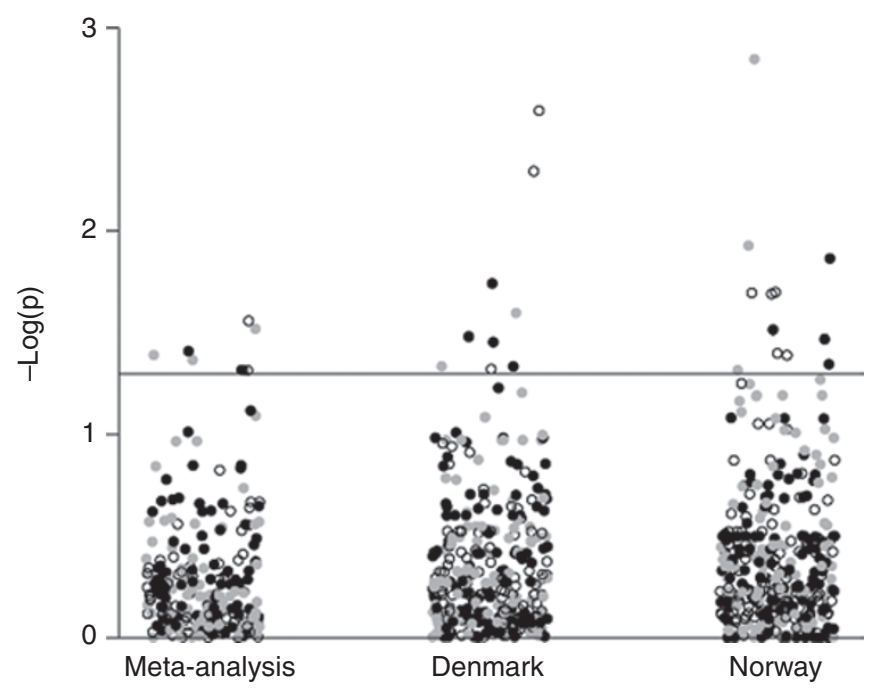

Figure 1. Significance of all tested SNPs. The significance, $-\log (p)$, of tests performed is represented graphically. Tests are stratified by the population (Denmark and Norway) and the pooled meta-analysis. Black points indicate PTD, open circles indicate very PTD, and gray points indicate PPROM. The horizontal line on the figure corresponds to $P=0.05$. PPROM, preterm prelabor rupture of membranes; PTD, preterm delivery; SNP, single-nucleotide polymorphism.

The results from each study were meta-analyzed to screen for any potential candidate SNPs related to PTD.

\section{RESULTS}

Case and control numbers for each study population, Danish and Norwegian, concerning the outcomes of PTD, very PTD, and preterm prelabor rupture of membranes (PPROM) are summarized in Table 1. Mitochondrial SNPs were tested using the outcomes defined in these tables for each population and then pooled via random-effects meta-analysis. Figure 1 is a graphical representation of the results for the Denmark and Norway populations and meta-analyzed results.

In the Danish population, 9 SNPs ( 4 for PTD, 3 for very PTD, and 2 for PPROM) fell below $P \leq 0.05$ and in the Norwegian population 12 SNPs were below $P \leq 0.05$ ( 4 for PTD, 5 for very PTD, and 3 for PPROM). These results can be seen in Tables 2 and 3 . None of these SNPs were below $P \leq 0.05$ in both populations and none approached the study-wide significance threshold $(P \leq 0.0006)$.

In the meta-analyzed results, for the outcome of PTD, there were a total of four SNPs that were below $P \leq 0.10$ (Table 4). Of these, two SNPs were below $P \leq 0.05$ at positions 6777 and
5047. None of these SNPs were below $P \leq 0.05$ in either the Danish or Norwegian populations alone and none approached study-wide significance $(P \leq 0.0006)$.

Results were similar when using the outcome of very PTD. After meta-analysis, three SNPs had $P \leq 0.10$ and all were below $P \leq 0.05$. Of these three, one was below $P \leq 0.05$ in the Danish population and two were below $P \leq 0.05$ in the Norwegian population. None approached study-wide significance $(P \leq 0.0006)$.

For the outcome of PPROM, four SNPs were below $P \leq 0.10$ and three were below $P \leq 0.05$. None of these SNPs were below $P \leq 0.05$ in either the Danish or Norwegian populations and none approached study-wide significance $(P \leq 0.0006)$.

No individual SNP, in the Danish cohort, Norwegian cohort, or pooled meta-analysis was even on the same order of magnitude of the study-wide significance threshold, $P \leq 0.0006$. Complete results for all tested SNPs are available from the authors on request.

\section{DISCUSSION}

Our analysis of mitochondrial SNPs and their relation with PTD and related outcomes did not implicate any particular locus that was significantly associated with any of the defined outcomes. This study does not support the hypothesis of a maternal link to PTD through transmission of the mitochondrial genome.

Assessing the mitochondrial impact on PTD is a necessary step in unraveling its etiology. In addition to epidemiological evidence for a mitochondrial role in PTD, biological factors point to this possibility. Oxidative stress from natural processes, both pregnancy (28) and labor (29), as well as outside factors like toxins (30) and infection, can contribute to adverse pregnancy outcomes and would be worsened in the setting of mitochondrial dysfunction. Similarly, given the central role of the mitochondrion in energy metabolism (31), variations in necessary processes, from mother or fetus, could lead to detrimental effects.

Mitochondrial genetics was an obvious explanation for the observed maternal link to PTD risk, but this may be explained by other modes of transmission. Genetically, the possibility of imprinting, whereby the maternal or paternal copy of a gene is expressed over the suppressed paternal or maternal copy, at key locations related to pregnancy and parturition may explain the observed inheritance pattern. Imprinting appears to be rare (32) and its impact in complex and common diseases is uncertain, but it has been shown to possibly contribute to other common conditions (33). Alterations in the maternal nuclear genome or fetal-maternal genetic interactions could also explain the witnessed maternal inheritance. Finally, any risk factor for PTD that is preferentially passed from mother to daughter, as opposed from mother to son, could explain this association. Such possibilities include biological factors such as genital tract microflora (34), or, conceivably, nongenetic transmission of response to social factors such as stress (35).

This study using mitochondrial genetics did have some limitations. First, although every effort was made to include 
Table 2. SNPs in the Danish population with $P<0.05$

\begin{tabular}{|c|c|c|c|c|c|c|}
\hline Location & $\begin{array}{c}\text { Random effects, } \\
P \text { value }\end{array}$ & $\begin{array}{l}\text { Random } \\
\text { effects, OR }\end{array}$ & $\begin{array}{l}P \text { value, } \\
\text { Denmark }\end{array}$ & $\begin{array}{c}\text { OR, } \\
\text { Denmark }\end{array}$ & $\begin{array}{l}P \text { value, } \\
\text { Norway }\end{array}$ & $\begin{array}{l}\text { OR, } \\
\text { Norway }\end{array}$ \\
\hline \multicolumn{7}{|l|}{ PTD } \\
\hline 8870 & $-^{a}$ & $--^{a}$ & 0.033 & $--^{a}$ & 0.316 & 3.01 \\
\hline 9378 & 0.325 & 0.50 & 0.035 & 0.21 & 0.809 & 0.89 \\
\hline 13106 & 0.028 & 3.39 & 0.003 & 4.67 & 0.807 & 1.30 \\
\hline 8870 & $-^{\mathrm{a}}$ & $-^{\mathrm{a}}$ & 0.005 & $-^{a}$ & 0.756 & 0.00 \\
\hline 2485 & $-^{\mathrm{a}}$ & $--^{\mathrm{a}}$ & 0.048 & $-^{a}$ & $-c$ & $-^{c}$ \\
\hline \multicolumn{7}{|l|}{ PPROM } \\
\hline
\end{tabular}

MAF, minor allele frequency; OR, odds ratio; PPROM, preterm prelabor rupture of membranes; PTD, preterm delivery; SNP, single-nucleotide polymorphism.

aln the Danish population, the controls had a MAF $=0$ for this SNP. Therefore, the OR and meta-analysis could not be evaluated. ${ }^{\circ} \mathrm{n}$ the Danish population, the cases had a MAF $=0$

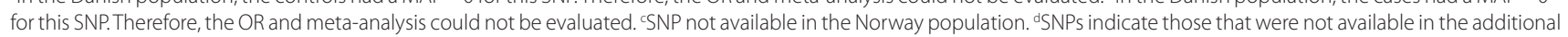
500 Danish samples. The meta-analysis results are based on the original Danish population and the Norwegian population.

Table 3. SNPs in the Norwegian population with $P<0.05$

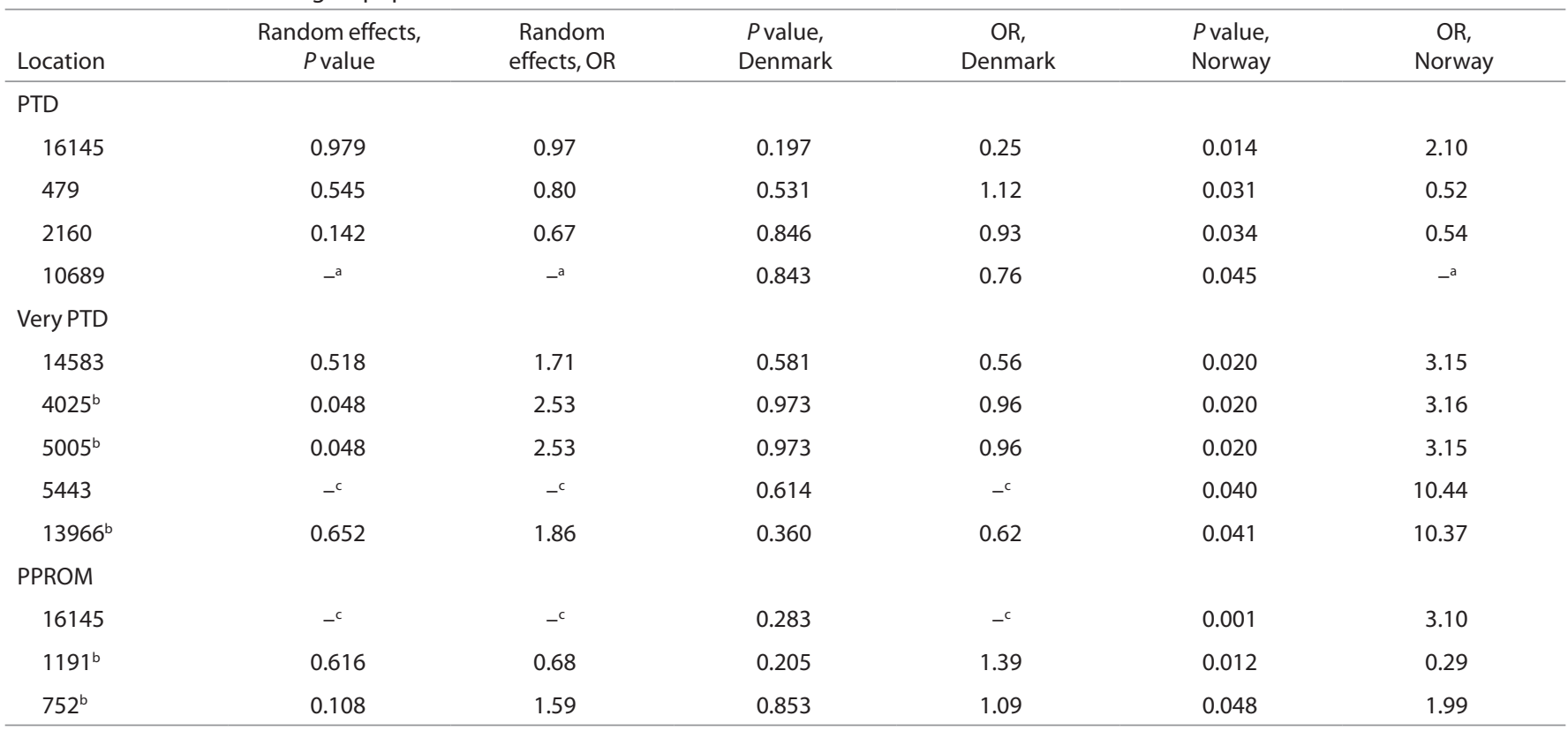

MAF, minor allele frequency; OR, odds ratio; PPROM, preterm prelabor rupture of membranes; PTD, preterm delivery; SNP, single-nucleotide polymorphism.

aln the Norwegian population, the controls had a MAF = 0 for this SNP. Therefore, the OR and meta-analysis could not be evaluated. ${ }^{b S N P s}$ indicate those that were not available in the additional 500 Danish samples. The meta-analysis results are based on the original Danish population and the Norwegian population. In the Danish population, the cases had a MAF $=0$ for this SNP. Therefore, the OR and meta-analysis could not be evaluated.

only spontaneous PTDs, it is possible that our case population included a small portion of PTDs resulting from infections. In the original Danish cohort, 12 cases $(<1.5 \%)$ had documented chorioamnionitis. We believe that the additional Danish samples and the Norwegian population have similarly low infection rates, and thus our case population is appropriately homogeneous. In addition, predefined haplogroups ( $\mathrm{H}, \mathrm{U}$, J, etc., for Western Europeans) were not tested because the Illumina chip did not contain the necessary SNPs to define these haplogroups in the populations. Meta-analysis of two study populations increased our power to detect any potential associated SNPs, but our study was still limited in its ability to detect the effects of rare variations. A sample size of around 5,000 cases and controls would be needed to detect an odds ratio of 2.0 for SNPs with minor allele frequency around $1 \%$. This is especially true for the outcomes of PPROM and very PTD because of the significant decrease in the case population. Studies of the current size can only 
Table 4. Nominally significant SNPs after meta-analysis for PTD, very PTD, and PPROM

\begin{tabular}{|c|c|c|c|c|c|c|}
\hline Location & $\begin{array}{c}\text { Random effects, } \\
P \text { value }\end{array}$ & $\begin{array}{l}\text { Random } \\
\text { effects, OR }\end{array}$ & $\begin{array}{l}P \text { value, } \\
\text { Denmark }\end{array}$ & $\begin{array}{c}\text { OR, } \\
\text { Denmark }\end{array}$ & $\begin{array}{l}P \text { value, } \\
\text { Norway }\end{array}$ & $\begin{array}{c}\text { OR, } \\
\text { Norway }\end{array}$ \\
\hline \multicolumn{7}{|l|}{ PTD } \\
\hline 5047 & 0.048 & 1.73 & 0.059 & 1.94 & 0.388 & 1.45 \\
\hline $6777^{a}$ & 0.039 & 0.69 & 0.143 & 0.67 & 0.139 & 0.71 \\
\hline 3916 & 0.076 & 0.73 & 0.207 & 0.75 & 0.203 & 0.69 \\
\hline 16146 & 0.097 & 0.78 & 0.335 & 0.81 & 0.171 & 0.76 \\
\hline \multicolumn{7}{|l|}{ Very PTD } \\
\hline 13106 & 0.028 & 3.39 & 0.003 & 4.67 & 0.807 & 1.30 \\
\hline $4025^{\mathrm{a}}$ & 0.048 & 2.53 & 0.973 & 0.96 & 0.020 & 3.16 \\
\hline $5005^{a}$ & 0.048 & 2.53 & 0.973 & 0.96 & 0.020 & 3.15 \\
\hline \multicolumn{7}{|l|}{ PPROM } \\
\hline 13106 & 0.030 & 2.41 & 0.188 & 2.19 & 0.068 & 2.60 \\
\hline 5047 & 0.041 & 2.06 & 0.082 & 2.16 & 0.239 & 1.92 \\
\hline $5657^{a}$ & 0.043 & 1.79 & 0.133 & 2.56 & 0.120 & 1.64 \\
\hline 15905 & 0.081 & 1.52 & 0.062 & 1.80 & 0.573 & 1.22 \\
\hline
\end{tabular}

OR, odds ratio; PPROM, preterm prelabor rupture of membranes; PTD, preterm delivery; SNP, single-nucleotide polymorphism.

aSNPs indicate those that were not available in the additional 500 Danish samples. These results are based on a meta-analysis between the original Danish population and the Norwegian population.

detect common SNP variations with large disease risk or uncommon SNP variations with extreme impact on disease. Traditionally, it has been thought that common SNP variations lead to common diseases, but this may not be true for all conditions (36). Therefore, only future studies with very large sample sizes and more granular sequencing can completely assess the impact of rare variations in the mitochondrial genome on the risk of PTD.

This study has several strengths that contribute to its validity. The data come from two populations that are genetically homogeneous, reducing population stratification and possible false positives. However, this may also limit the application of these results to other external populations. Definitions of PTD were clearly established for cases and controls, as they were based on last menstrual period and/or ultrasound. The case and control groups were both clean of many possible confounding conditions including any induction of delivery, multiple births, congenital malformations, preeclampsia, or other placental abnormalities.

Previous epidemiological and genetic studies indicate that genetics plays some role in the etiology of PTD. Future studies should include analyses of the effects of imprinting; a full genome-wide approach is currently under way within these same populations. Extending studies of mitochondrial genetics in a larger cohort of this population or in different ethnic populations may yield contrasting results and gene-gene and gene-environment interactions between factors such as inflammation and cholesterol are of interest. Genetic interactions in the mother and/or fetus likely play a role in the etiology of PTD. However, no obvious common mitochondrial variant was associated with any of the study outcomes in these populations.

\section{METHODS}

\section{Study Populations}

Danish Population. Mother-neonate pairs were selected as cases and controls from the Danish National Birth Cohort (DNBC) Study (37). This cohort consisted of $\sim 100,000$ total births occurring between 1997 and 2002. Controls were defined as 39-40 wk GA and cases as infants between 20 and 36 wk GA. (Only three births were $<22$ wk GA. As only live-born infants were included in the study, these infants were unlikely survivors, had underestimated GA, or had a data error in their stillborn status. Underestimated GA is most likely; therefore, they have been retained in the cohort.) All four grandparents of a neonate were born in Denmark, except for 24 individuals who had one or two grandparents born in other Nordic countries. The parent genome-wide association study included 1,000 case mother-neonate pairs and 1,000 control mother-neonate pairs. Biological samples collected from mothers and neonates were stored in the Biobank at Statens Serum Institut. Of the original 2,000 mothers chosen, 1,937 were successfully genotyped. Of these, 41 mothers were removed for having GA outside the case or control definitions, 165 cases for delivery via cesarean section, 24 controls for having a previous PTD, and 2 mothers for preeclampsia. In addition, after genotyping, some mothers were identified as sisters, therefore five women were removed via random selection of one sister-neonate pair. This left 1,700 mothers for analysis (965 controls and 735 cases). Of the original 2,000 neonates chosen, 1,901 were successfully genotyped. Of these, 47 were removed for GA not within the case or control definition, 154 cases for delivery via cesarean section, 23 controls for their mothers having a previous PTD, and 2 for preeclampsia. In addition, four neonates were removed for being born to mothers who were sisters to others in the study. This left 1,671 neonates for analysis (958 controls and 713 cases).

To increase the power for SNP detection for the original genomewide association study described above, an additional set of $\sim 500$ mother-baby pairs, mostly cases, were selected from the DNBC BioBank or the Danish Newborn Screening Biobank. Of these 500 mothers, 496 were successfully genotyped and 2 were removed for GA not within the case or control range, leaving 490 cases and 4 controls. Of the 500 neonates, all were successfully genotyped and 2 were removed for GA not within the case or control range, leaving 494 cases and 4 controls. 
Norwegian Population. Mother-neonate pairs were selected from the Norwegian Mother and Child Cohort Study (MoBa) conducted by the Norwegian Institute of Public Health (38). MoBa is a population-based prospective pregnancy cohort including 108,000 children, 90,700 mothers, and 71,500 fathers recruited from all over Norway from 1999 to 2008. Each participant had to give a written informed consent upon recruitment, and the participation rate was 38.5\% among invited women. Blood samples were collected from both parents at the routine ultrasound examination in week 17-18 of pregnancy and from mothers and children at delivery. Patient samples were stored at the Biobank of the Norwegian Institute of Public Health (39). Epidemiological data were obtained through questionnaires given at regular intervals and linkage to the Medical Birth Registry of Norway. Our study is based on version 4 of the quality-assured data files released for research in 2008.

Controls were selected from infants born at 39-40wk gestation and cases were those who were born from 22 to $36 \mathrm{wk}$ gestation. Only mothers who delivered between 20 and 34 y of age were eligible for selection. Women with a medical history of diabetes, chronic hypertension, autoimmune diseases like systemic lupus erythematosus, scleroderma, rheumatoid arthritis, inflammatory bowel disease, or immunocompromising diseases were excluded. Women with gestational diabetes, hypertension in pregnancy or cervical cerclage; pregnancies conceived by in vitro fertilization; stillbirths; and neonates who were small or large for GA were also excluded. Before quality control, 1,921 mothers (951 cases and 970 controls) were selected for genotyping. Of these, 81 were removed for low genotyping rate, 6 for unknown sex, and 13 for nonrelatedness to the assigned child. In addition, 19 control mothers were excluded for having a previous PTD, leaving 1,802 individuals successfully genotyped (903 cases and 899 controls). There were 1,200 children available for genotyping (582 cases and 618 controls). Of these, 42 were removed for low genotyping and 6 for nonrelatedness to the assigned mother. Furthermore, 15 control children were excluded for having a mother with a previous PTD, leaving 1,137 children for further analysis (556 cases and 581 controls). Fewer children than mothers were genotyped due to lack of available DNA.

\section{Common Inclusion-Exclusion Criteria}

Exclusion criteria for each study were any obstetrical induction of labor (iatrogenic delivery), placental abnormalities (previa or abruption), preeclampsia, congenital malformations, stillbirths, and multiple births. All cesarean sections were removed from the case populations as well. Subjects were enrolled with the appropriate consent for future research. In both the Danish and Norwegian cohorts, the number of eligible births for this study represented $\sim 25 \%$ of the total PTD population. Informed consent was obtained from all individuals according to each country's protocols $(37,38)$ and approval for this study was granted by the University of Iowa Institutional Review Board, study no. 200608748.

\section{Genotyping}

Genotyping was performed using the Illumina Human660WQuadv1_A (Illumina, San Diego, CA), which contained 135 SNPs assumed to have minor allele frequency $>1 \%$ in Caucasian populations based on public databases (http://www.mitomap.org). This Illumina chip uses the HapMap Yoruba sample (accession number AF347015) as a reference. The standard reference for mitochondrial SNPs is the revised Cambridge Reference Sequence (accession no. NC_012920). Positions reported in this study are according to the AF347015 sequence. The conversion from AF347015 to NC_012920 can be made using the map located at this website: http://www.mitomap.org/Sandbox/ YorubanConversion. In total, 88 SNPs were meta-analyzed between the two populations. For the Danish samples, the call rate was $>99 \%$ for all included meta-analyzed SNPs and the median minor allele frequency was 0.025 . For the Norwegian population, the call rate was also $>99 \%$ and the median minor allele frequency was 0.024 . For the additional 500 Danish samples, only 109 SNPs were called during genotyping.

\section{Union of Maternal and Neonatal Genetic Information}

For mitochondrial data, mothers and neonates are assumed to have identical genotypes. The genetic concordance rate between maternal and neonatal pairs was $100 \%$ for all SNPs in the Danish population and $>99.4 \%$ in the Norway population. Therefore, maternal and neonatal genetic information was analyzed as one individual, using the mother's genotype as the default and supplementing with the neonate's genotype if that of the mother was missing. This increased the power of the individual studies by increasing genotyping rates and increasing the number of cases and controls. Table 1 indicates the number of case and control genotypes available to be analyzed for each of the populations.

\section{Genetic Analysis}

Single SNP allelic associations were tested using $\chi^{2}$ tests in both the Danish and Norwegian populations. The outcomes examined were PTD ( $\leq 36 \mathrm{wk}$ GA), very PTD ( $\leq 32 \mathrm{wk}$ GA), and PPROM. PPROM was defined by using the International Classification of Diseases (ICD) code in the Medical Birth Register for each country. After testing within each population, the results were meta-analyzed using a random-effects model. No correction for the number of tests was made to the reported pooled $P$ values or odds ratios. All analyses were performed in PLINK (40).

Of the 135 mitochondrial SNPs on the panel, a total of 88 SNPs were meta-analyzed between the two populations. The 47 SNPs removed were either not called in one of the populations or had a minor allele frequency of zero in either the cases or controls for one of the populations. For the additional 500 Danish cases and controls, only 109 SNPs were called. Given the number of SNPs tested, a conservative threshold for significance in this study would be $P=0.0006$ $(P=0.05 / 88$ SNPs).

\section{ACKNOWLEDGMENTS}

We thank Teri Manolio, who was instrumental in the completion of this genome-wide association study. In addition, we thank Hakon K. Gjessing for useful comments and discussion.

\section{STATEMENT OF FINANCIAL SUPPORT}

This work was supported by the National Institutes of Health (NIH) (grants HD52953 and HD57192 to J.C.M.). The Danish National Birth Cohort (DNBC) was established with the support of a major grant from the Danish National Research Foundation. Additional support for the DNBC has been provided by the Danish Pharmacists' Fund, the Egmont Foundation, the March of Dimes Birth Defects Foundation, the Augustinus Foundation, and the Health Fund of the Danish Health Insurance Societies. The generation of genome-wide association study genotype data for the DNBC samples was funded as part of the Gene Environment Association Studies (GENEVA) under the Genes, Environment, and Health Initiative (GEI). Assistance with phenotype harmonization and genotype cleaning, as well as with general study coordination, was provided by the GENEVA Coordinating Center (U01 HG004446). Assistance with data cleaning was provided by the National Center for Biotechnology Information. Funding support for genotyping, which was performed at the Johns Hopkins University Center for Inherited Disease Research (CIDR) and the Broad Institute of MIT and Harvard, was provided by the NIH GEI (U01HG004438 for CIDR; U01HG04424 for Broad Institute) and the NIH contract "High throughput genotyping for studying the genetic contributions to human disease" (HHSN268200782096C for CIDR only). The Norwegian Mother and Child Cohort Study is supported by the Norwegian Ministry of Health and the Ministry of Education and Research, NIH/National Institute of Environmental Health Sciences (NIEHS) (contract NO-ES-75558), NIH/National Institute of Neurological Disorders and Stroke (NINDS) (grant 1 UO1 NS 047537-01), and the Norwegian Research Council/Research in Functional Genomics in Norway (FUGE) (grant 151918/S10). The study was approved by the Regional Committee for Medical Research Ethics in South-Eastern Norway (S-06075) and the Norwegian Data Inspectorate (05/016784). Support also came from the Norwegian Research Council (FUGE 183220/S10, FRIMEDKLI-05 ES236011), Swedish government grants to researchers in the public health service (ALF) (ALFGBG-136431), Sahlgrenska University Hospital and Sahlgrenska Academy (Gothenburg, Sweden), the Swedish Medical Society (Stockholm, Sweden; 2008-21198), and the Jane and Dan Olsson Research Foundation (Gothenburg, Sweden). The MoBa samples were genotyped at the genotyping core facility at Oslo University Hospital (Norway).

Disclosure: The authors declared no conflict of interest. 


\section{REFERENCES}

1. Callaghan WM, MacDorman MF, Rasmussen SA, Qin C, Lackritz EM. The contribution of preterm birth to infant mortality rates in the United States. Pediatrics 2006;118:1566-73.

2. Lawn JE, Cousens S, Zupan J; Lancet Neonatal Survival Steering Team. 4 million neonatal deaths: when? Where? Why? Lancet 2005;365:891-900.

3. Marlow N, Wolke D, Bracewell MA, Samara M; EPICure Study Group. Neurologic and developmental disability at six years of age after extremely preterm birth. N Engl J Med 2005;352:9-19.

4. Wood NS, Marlow N, Costeloe K, Gibson AT, Wilkinson AR. Neurologic and developmental disability after extremely preterm birth. EPICure Study Group. N Engl J Med 2000;343:378-84.

5. Martin JA, Hamilton BE, Sutton PD, Ventura SJ, Mathews TJ, Osterman MJ. Births: final data for 2008. Natl Vital Stat Rep 2010;59:1, 3-71.

6. Cnattingius $\mathrm{S}$. The epidemiology of smoking during pregnancy: smoking prevalence, maternal characteristics, and pregnancy outcomes. Nicotine Tob Res 2004;6:Suppl 2:S125-40.

7. Thompson JM, Irgens LM, Rasmussen S, Daltveit AK. Secular trends in socio-economic status and the implications for preterm birth. Paediatr Perinat Epidemiol 2006;20:182-7.

8. Dole N, Savitz DA, Hertz-Picciotto I, Siega-Riz AM, McMahon MJ, Buekens P. Maternal stress and preterm birth. Am J Epidemiol 2003;157:14-24.

9. Fraser AM, Brockert JE, Ward RH. Association of young maternal age with adverse reproductive outcomes. N Engl J Med 1995;332:1113-7.

10. Jacobsson B, Ladfors L, Milsom I. Advanced maternal age and adverse perinatal outcome. Obstet Gynecol 2004;104:727-33.

11. Mercer BM, Goldenberg RL, Moawad AH, et al. The preterm prediction study: effect of gestational age and cause of preterm birth on subsequent obstetric outcome. National Institute of Child Health and Human Development Maternal-Fetal Medicine Units Network. Am J Obstet Gynecol 1999;181(5 Pt 1):1216-21.

12. Jakobsson M, Gissler M, Sainio S, Paavonen J, Tapper AM. Preterm delivery after surgical treatment for cervical intraepithelial neoplasia. Obstet Gynecol 2007;109(2 Pt 1):309-13.

13. Edison RJ, Berg K, Remaley A, et al. Adverse birth outcome among mothers with low serum cholesterol. Pediatrics 2007;120:723-33.

14. Bodnar LM, Catov JM, Simhan HN, Holick MF, Powers RW, Roberts JM. Maternal vitamin D deficiency increases the risk of preeclampsia. J Clin Endocrinol Metab 2007;92:3517-22.

15. Bodnar LM, Krohn MA, Simhan HN. Maternal vitamin D deficiency is associated with bacterial vaginosis in the first trimester of pregnancy. J Nutr 2009;139:1157-61.

16. Goldenberg RL, Hauth JC, Andrews WW. Intrauterine infection and preterm delivery. N Engl J Med 2000;342:1500-7.

17. Romero R, Espinoza J, Gonçalves LF, Kusanovic JP, Friel LA, Nien JK. Inflammation in preterm and term labour and delivery. Semin Fetal Neonatal Med 2006;11:317-26.

18. Muglia LJ, Katz M. The enigma of spontaneous preterm birth. N Engl J Med 2010;362:529-35.

19. Kistka ZA, DeFranco EA, Ligthart L, et al. Heritability of parturition timing: an extended twin design analysis. Am J Obstet Gynecol 2008;199:43. e1-5.

20. Crider KS, Whitehead N, Buus RM. Genetic variation associated with preterm birth: a HuGE review. Genet Med 2005;7:593-604.
21. Engel SA, Erichsen HC, Savitz DA, Thorp J, Chanock SJ, Olshan AF. Risk of spontaneous preterm birth is associated with common proinflammatory cytokine polymorphisms. Epidemiology 2005;16:469-77.

22. Menon R, Velez DR, Simhan H, et al. Multilocus interactions at maternal tumor necrosis factor-alpha, tumor necrosis factor receptors, interleukin-6 and interleukin-6 receptor genes predict spontaneous preterm labor in European-American women. Am J Obstet Gynecol 2006;194:1616-24.

23. Boyd HA, Poulsen G, Wohlfahrt J, Murray JC, Feenstra B, Melbye M. Maternal contributions to preterm delivery. Am J Epidemiol 2009;170:1358-64.

24. Svensson AC, Sandin S, Cnattingius S, et al. Maternal effects for preterm birth: a genetic epidemiologic study of 630,000 families. Am J Epidemiol 2009;170:1365-72.

25. Lunde A, Melve KK, Gjessing HK, Skjaerven R, Irgens LM. Genetic and environmental influences on birth weight, birth length, head circumference, and gestational age by use of population-based parent-offspring data. Am J Epidemiol 2007;165:734-41.

26. Velez DR, Menon R, Simhan H, Fortunato S, Canter JA, Williams SM. Mitochondrial DNA variant A4917G, smoking and spontaneous preterm birth. Mitochondrion 2008;8:130-5.

27. Goldenberg RL, Culhane JF, Iams JD, Romero R. Epidemiology and causes of preterm birth. Lancet 2008;371:75-84.

28. Myatt L, Cui X. Oxidative stress in the placenta. Histochem Cell Biol 2004;122:369-82.

29. Fainaru O, Almog B, Pinchuk I, Kupferminc MJ, Lichtenberg D, Many A. Active labour is associated with increased oxidisibility of serum lipids ex vivo. BJOG 2002;109:938-41.

30. Mohorovic L. First two months of pregnancy-critical time for preterm delivery and low birthweight caused by adverse effects of coal combustion toxics. Early Hum Dev 2004;80:115-23.

31. Xu Y, Wang Q, Cook TJ, Knipp GT. Effect of placental fatty acid metabolism and regulation by peroxisome proliferator activated receptor on pregnancy and fetal outcomes. J Pharm Sci 2007;96:2582-606.

32. Morison IM, Ramsay JP, Spencer HG. A census of mammalian imprinting. Trends Genet 2005;21:457-65.

33. Cui H, Cruz-Correa M, Giardiello FM, et al. Loss of IGF2 imprinting: a potential marker of colorectal cancer risk. Science 2003;299:1753-5.

34. Guaschino S, De Seta F, Piccoli M, Maso G, Alberico S. Aetiology of preterm labour: bacterial vaginosis. BJOG 2006;113:Suppl 3:46-51.

35. Caldji C, Diorio J, Meaney MJ. Variations in maternal care in infancy regulate the development of stress reactivity. Biol Psychiatry 2000;48: 1164-74.

36. Manolio TA, Collins FS, Cox NJ, et al. Finding the missing heritability of complex diseases. Nature 2009;461:747-53.

37. Olsen J, Melbye M, Olsen SF, et al. The Danish National Birth Cohort-its background, structure and aim. Scand J Public Health 2001;29:300-7.

38. Magnus P, Irgens LM, Haug K, Nystad W, Skjaerven R, Stoltenberg C; MoBa Study Group. Cohort profile: the Norwegian Mother and Child Cohort Study (MoBa). Int J Epidemiol 2006;35:1146-50.

39. Rønningen KS, Paltiel L, Meltzer HM, et al. The biobank of the Norwegian Mother and Child Cohort Study: a resource for the next 100 years. Eur J Epidemiol 2006;21:619-25.

40. Purcell S, Neale B, Todd-Brown K, et al. PLINK: a tool set for wholegenome association and population-based linkage analyses. Am J Hum Genet 2007;81:559-75. 